\title{
Synthesis and Biological Evaluation of Novel Jatrorrhizine Derivatives with Amino Groups Linked at the 3-Position as Inhibitors of Acetylcholinesterase
}

\author{
Xiaofei Jiang, ${ }^{1,2,3}$ Nengling Zhang, ${ }^{4}$ Nanqian Xiong, ${ }^{2}$ Yi Liu, ${ }^{2}$ \\ Jianfeng Cao, ${ }^{2}$ and ShengXiang Qiu ${ }^{1}$ \\ ${ }^{1}$ Key Laboratory of Plant Resources Conservation and Sustainable Utilization, South China Botanical Garden, \\ Chinese Academy of Sciences, Guangzhou 510006, China \\ ${ }^{2}$ School of Chemistry and Life Sciences, Guizhou Education University, Guiyang 550018, China \\ ${ }^{3}$ College of Chemical and Environmental Engineering, Chongqing Three Gorges University, Chongqing 404000, China \\ ${ }^{4}$ School of Pharmacy, Guizhou Medical University, Guiyang 550025, China
}

Correspondence should be addressed to ShengXiang Qiu; sxqiu@scbg.ac.cn

Received 26 May 2017; Accepted 4 October 2017; Published 13 December 2017

Academic Editor: Henryk Kozlowski

Copyright (C) 2017 Xiaofei Jiang et al. This is an open access article distributed under the Creative Commons Attribution License, which permits unrestricted use, distribution, and reproduction in any medium, provided the original work is properly cited.

\begin{abstract}
Jatrorrhizine was considered as one of the active constituents of Coptis chinensis Franch. Herein, jatrorrhizine derivatives with substituted amino groups linked at the 3-position were designed, synthesized, and biologically evaluated as inhibitors of acetylcholinesterase. Jatrorrhizine derivatives inhibited the activity of acetylcholinesterase (AChE) to a greater extent than the lead compound jatrorrhizine. All these jatrorrhizine derivatives were proved to be potent inhibitors of acetylcholinesterase (AChE) with submicromolar $\mathrm{IC}_{50}$ values, but less sensitive to butyrylcholinesterase $(\mathrm{BuChE})$, which suggests that these jatrorrhizine derivatives are selective for $\mathrm{AChE} / \mathrm{BuChE}$. Compound $3 \mathrm{~g}$ gave the most potent inhibitor activity for $\mathrm{AChE}\left(\mathrm{IC}_{50}=0.301 \mu \mathrm{M}\right)$, which is greater than the lead compound jatrorrhizine. All these results demonstrated that these jatrorrhizine derivatives are potential inhibitors for AChE.
\end{abstract}

\section{Introduction}

Alzheimer's disease (AD) is the most prevalent form of dementia affecting approximately six million people in China [1]. One possible approach to treat $\mathrm{AD}$ is to restore the level of acetylcholine (ACh) by inhibiting acetylcholinesterase (AChE) with reversible inhibitors. The aim of AChE inhibitors is to improve the endogenous levels of ACh in the brain of $\mathrm{AD}$ patients, thereby increasing cholinergic neurotransmission [2]. Rhizoma Coptidis can be used for treatment of cardiovascular and neurodegenerative diseases [3]; Coptis chinensis Franch extracts show good inhibitory activity for $\mathrm{AChE}$ in vitro [4]. Jatrorrhizine is a kind of quaternary protoberberine alkaloids (QPA) and was considered as one of the active constituents of Coptis chinensis Franch $[5,6]$. Jatrorrhizine has multiple bioactivities, such as hypoglycemic [7], antimicrobial [8], and antioxidant activities [9]. In recent years, berberine derivatives with various heterocyclic rings, such as thiophene, pyrrole, piperidine, and carbazole, were demonstrated as both acetylcholinesterase (AChE) and butyrylcholinesterase (BuChE) inhibitors [10-12]. However, no literature reported that jatrorrhizine and its derivatives could be used as inhibitors of both AChE and BuChE. Taking into account the fact that jatrorrhizine and berberine belong to quaternary protoberberine alkaloid (QPA), we report the design, synthesis, and biological evaluation of a series of novel jatrorrhizine derivatives which have an amino group linked at the 3-position as both $\mathrm{AChE}$ and BuChE inhibitors in this paper, hoping that these derivatives could be used as agents for Alzheimer's disease (AD).

\section{Results and Discussion}

The spectra data and other characterization of the target compounds $\mathbf{3 a - 3 f}$ are shown in the experimental section. The yield of the derivatives was ranged from $59 \%$ to $41 \%$. 
TABLE 1: In vitro inhibition $\mathrm{IC}_{50}(\mu \mathrm{M})$ and selectivity of jatrorrhizine derivatives for $\mathrm{AChE}$ and BuChE.

\begin{tabular}{lccc}
\hline Compounds & $\mathrm{AChE}^{\mathrm{a}}$ & $\mathrm{IC}_{50}(\mu \mathrm{M})$ & BuChE $^{\mathrm{b}}$ \\
\hline Jatrorrhizine & $0.872 \pm 0.87$ & $>100$ & $>115$ \\
Tacrine & $0.223 \pm 0.003$ & $0.052 \pm 0.009$ & 0.23 \\
3a & $0.500 \pm 0.016$ & $>100$ & $>216$ \\
3b & $0.461 \pm 0.004$ & $>100$ & $>260$ \\
3c & $0.322 \pm 0.016$ & $>100$ & $>310$ \\
3d & $0.41 \pm 0.011$ & $>100$ & $>243$ \\
3e & $0.355 \pm 0.012$ & $>100$ & $>338$ \\
3f & $0.821 \pm 0.023$ & $>100$ & $>121$ \\
3g & $0.301 \pm 0.038$ & $>300$ & \\
\hline
\end{tabular}

${ }^{\mathrm{a}} 50 \%$ inhibitory concentration (means \pm SEM of three experiments) of AChE from electric eel; ${ }^{b} 50 \%$ inhibitory concentration (means \pm SEM of three experiments) of $\mathrm{BuChE}$ from equine serum; ${ }^{\mathrm{c}}$ selectivity for $\mathrm{AChE}=\mathrm{IC}_{50}(\mathrm{BuChE}) / \mathrm{IC}_{50}(\mathrm{AChE})$.

The spectra data of jatrorrhizine (1) was reported in [15]. The ${ }^{1} \mathrm{H}$ NMR spectrum of the target compounds showed that some new proton signals of $\mathrm{CH}_{2}$ and $\mathrm{CH}_{3}$ appeared at high field region. The compounds $3 \mathbf{a}-\mathbf{f}$ at $\delta 13-60$ area increased the signal of compound $\mathrm{C}$, the increased number of $\mathrm{C}$ is the same as $\mathrm{C}$ of substituent group switching on at the 3-position of jatrorrhizine. MS tested compounds formula. Both analytical and spectral data of all the newly synthesized compounds are in full agreement with the proposed structures.

The $\mathrm{IC}_{50}$ values for $\mathrm{AChE}$ and $\mathrm{BuChE}$ inhibition are shown in Table 1. All the jatrorrhizine derivatives demonstrated potent inhibitory activity against AChE with submicromolar $\mathrm{IC}_{50}$ values. The optimal AChE inhibition potency $\left(\mathrm{IC}_{50}=0.301 \mu \mathrm{M}\right)$ was provided by compound $3 \mathrm{~g}$; the inhibition activity of jatrorrhizine derivatives to $\mathrm{BuChE}$ is lower than AChE. It is interesting that all of the jatrorrhizine derivatives exhibited potent inhibitory activity for AChE compared to jatrorrhizine. The volume of the substituted amino groups has important impact on inhibitory activity. Among all the jatrorrhizine derivatives, the amino group gave the best results $\left(\mathrm{IC}_{50}=0.301 \mu \mathrm{M}\right)$. Interestingly, the cyclic substituted amino groups appeared to have weak activity compared to chain substituted amino groups. For example, compound $\mathbf{3 g}$ showed the highest inhibitory activity. This result indicated that the groups at the end of the molecule influenced inhibitory activity. In vitro, BuChE inhibition was also determined using the same method. The jatrorrhizine and jatrorrhizine derivatives demonstrated poor inhibitory potency against BuChE. These results proved that the jatrorrhizine derivatives have good selectivity for AChE and BuChE (Table 1).

\section{Experimental Section}

3.1. Chemistry. Jatrorrhizine was extracted and purified from Coptis chinensis Franch according to [16]. The purity of jatrorrhizine was up to $98 \%$ by HPLC. All of the other reagents were AR grade, purchased from Shanghai Aladdin Bio-Chem Technology Co., Ltd. Melting points were determined on an $\mathrm{RD}-2 \mathrm{C}$ electrothermal melting point apparatus and are uncorrected. The ${ }^{1} \mathrm{H}$ and ${ }^{13} \mathrm{C}$ NMR spectra were recorded on
Bruker $400(400 \mathrm{MHz})$ using TMS as the internal standard and $\mathrm{CD}_{3} \mathrm{OD}$ as solvent. Mass spectrometry (MS) spectra were collected on 1100 series LC/MSD instrument. The separation of the compounds was performed on silica gel-GF254 thin layers, with a moving phase of $\mathrm{C}_{6} \mathrm{H}_{6} / \mathrm{EtOAc} / \mathrm{MeOH} / \mathrm{i}$ $\mathrm{C}_{3} \mathrm{H}_{7} \mathrm{OH} / \mathrm{NH}_{3} \cdot \mathrm{H}_{2} \mathrm{O}(6: 3: 1.5: 1.5: 0.5)$.

3.2. Synthesis of Monomodified Jatrorrhizine Derivatives. Jatrorrhizine derivatives were synthesized according to [17]. The synthetic pathway of 3-substituted jatrorrhizine derivatives are shown in Figure 1. The alkylation of jatrorrhizine using 1,4-dibromoethane was conducted under the basic condition in $\mathrm{CH}_{3} \mathrm{CN}$ and afforded product 2 in $69 \%$ yield. $3 \mathbf{a}-\mathbf{3 f}$ were prepared by intermediates $2(0.1 \mathrm{~mol})$ with commercially available secondary amines $(0.11 \mathrm{~mol})$ (dimethylamine, pyrrolidine, etc.) in DMF, and additional alkaline catalyst $\left(\mathrm{K}_{2} \mathrm{CO}_{3}, 0.2 \mathrm{~mol}\right)$ was added, giving a $41-59 \%$ yield, respectively. Ammonolysis of jatrorrhizine $(0.05 \mathrm{~mol})$ with ammonia solution $(3 \mathrm{~mL})-\mathrm{NH}_{4} \mathrm{Cl}(0.05 \mathrm{~mol})$ in $\mathrm{CH}_{3} \mathrm{OH}$ at r.t. gave compounds 3 g. Heating the 2-iodoethanol $(0.01 \mathrm{mmol})$ and jatrorrhizine $(0.01 \mathrm{mmol})$ in $\mathrm{DMF}$ at $60^{\circ} \mathrm{C}$ would afford the desired product 3f. These compounds were added to reflux $\mathrm{MeOH}$ containing $\mathrm{AgCl}$ and converted into corresponding chlorides. All of the compounds were purified by chromatography on an $\mathrm{Al}_{2} \mathrm{O}_{3}$ column with $\mathrm{CH}_{3} \mathrm{OH} / \mathrm{CH}_{3} \mathrm{Cl}(9: 1)$ as eluent to give the target products (Figure 1).

Intermediates 2: 3-(4-Bromobutoxy)-2,9,10-trimethoxy-5,6-dihydroisoquinoli-no[3,2-a]isoquinolin-7-ium Chloride. Yield: 69\%, yellow solid; m.p.: 204-205 C. MS $(\mathrm{m} / z) 472.7[\mathrm{M}]^{+}$, $475.5[\mathrm{M}+2]^{+} .{ }^{1} \mathrm{H}$ NMR (400 MHz, CD $\left.\mathrm{OD}\right), \delta: 9.75$ (s, $1 \mathrm{H}$, $8-\mathrm{H}), 8.81$ (s, 1H, 13-H), 8.11 (d, $J=7.2 \mathrm{~Hz}, 1 \mathrm{H}, 11-\mathrm{H}), 8.07$ (d, $J=7.2 \mathrm{~Hz}, 1 \mathrm{H}, 12-\mathrm{H}), 7.67$ (s, 1H, 1-H), 7.01 (s, 1H, 4-H), $4.92(\mathrm{t}, J=6.8 \mathrm{~Hz}, 2 \mathrm{H}, 6-\mathrm{H}), 4.25\left(\mathrm{t}, J=6.8 \mathrm{~Hz}, 2 \mathrm{H},-\mathrm{CH}_{2}-\right)$, $4.15\left(\mathrm{~s}, 3 \mathrm{H},-\mathrm{OCH}_{3}-\right), 4.13\left(\mathrm{t}, \mathrm{J}=6.8 \mathrm{~Hz}, 2 \mathrm{H},-\mathrm{CH}_{2} \mathrm{O}-\right), 4.09(\mathrm{~s}$, $\left.3 \mathrm{H},-\mathrm{OCH}_{3}-\right), 4.06\left(\mathrm{~s}, 3 \mathrm{H},-\mathrm{OCH}_{3}-\right), 2.04-2.08\left(\mathrm{~m}, 2 \mathrm{H},-\mathrm{CH}_{2}-\right.$ ), $1.79-1.83\left(\mathrm{~m}, 2 \mathrm{H},-\mathrm{CH}_{2}-\right) .{ }^{13} \mathrm{C} \mathrm{NMR}\left(100 \mathrm{MHz}, \mathrm{CD}_{3} \mathrm{OD}\right) \delta$ : 153.0, 151.9, 151.0, 146.3, 145.7, 139.7, 135.2, 130.0, 128.0, 124.5, $123.3,121.3,120.5,113.2,110.0,70.0,62.5,57.6,57.4,57.0,47.7$, $29.3,27.8,26.8$. 


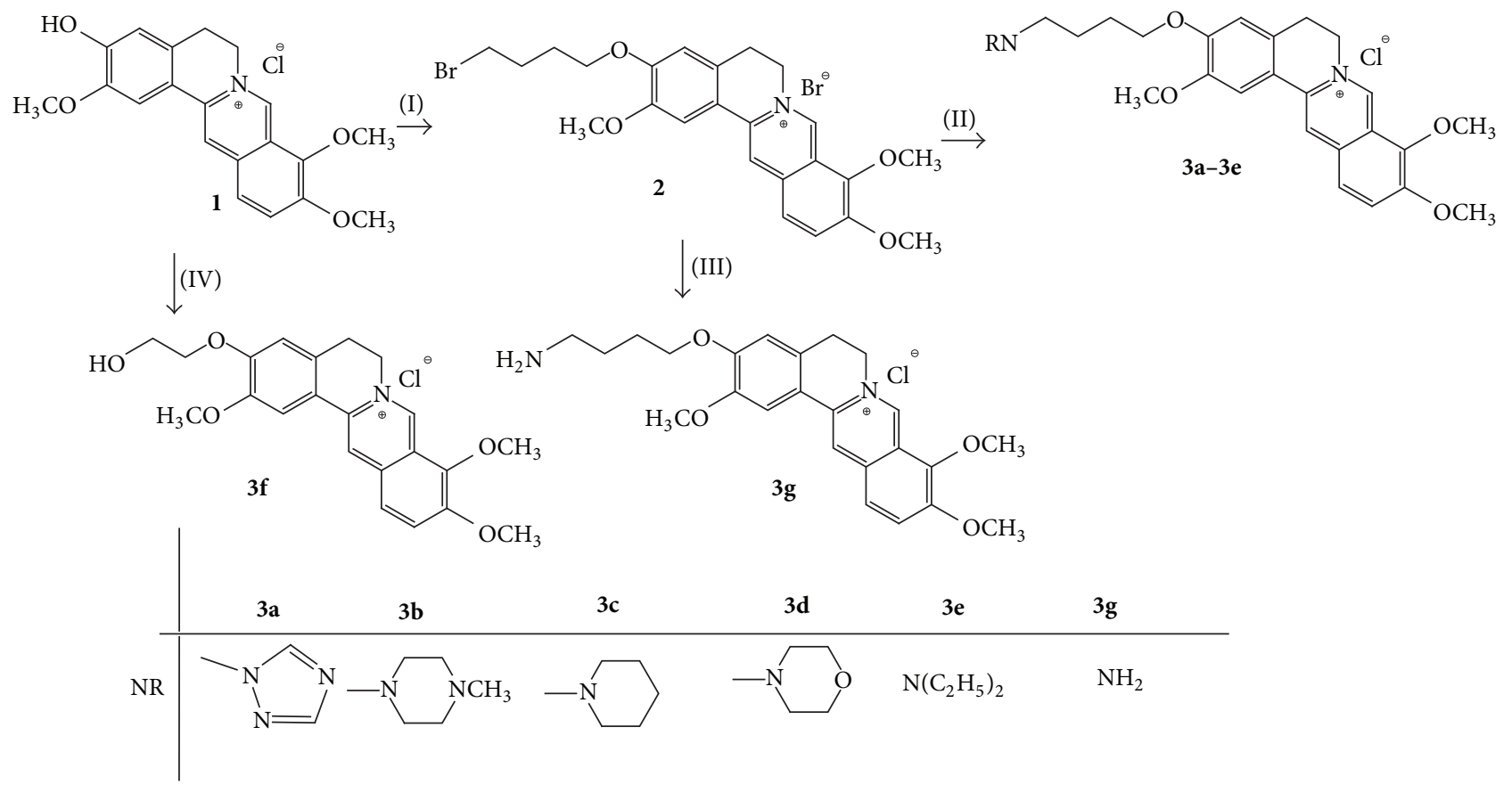

FIGURE 1: Synthetic route of 3-substituted jatrorrhizine derivatives (3a-3g).

3-(4-(4H-1,2,4-Triazol-4-yl)butoxy)-2,9,10-trimethoxy-5,6dihydroisoquinolino[3,2-a]isoquinolin-7-ium Chloride (3a). Yield: $46 \%$. yellow solid; m.p: $210-211^{\circ} \mathrm{C}$. MS $(\mathrm{m} / z) 461.3$ $[\mathrm{M}]^{+},{ }^{1} \mathrm{H}$ NMR $\left(400 \mathrm{MHz}, \mathrm{CD}_{3} \mathrm{OD}\right), \delta: 9.73$ (s, $\left.1 \mathrm{H}, 8-\mathrm{H}\right)$, 8.78 (s, 1H, 13-H), 8.57 (s, $1 \mathrm{H}$, Tri-H), 8.06 (d, $J=7.2 \mathrm{~Hz}, 1 \mathrm{H}$, 11-H), 8.00 (s, 1H, Tri 5-H), 8.01 (d, $J=7.2 \mathrm{~Hz}, 1 \mathrm{H}, 12-\mathrm{H})$, $7.61(\mathrm{~s}, 1 \mathrm{H}, 1-\mathrm{H}), 6.99(\mathrm{~s}, 1 \mathrm{H}, 4-\mathrm{H}), 4.92(\mathrm{t}, J=6.8 \mathrm{~Hz}, 2 \mathrm{H}$, $6-\mathrm{H}), 4.38$ (t, $J=6.8 \mathrm{~Hz}, 2 \mathrm{H}$, Tri-CH2), 4.19 (s, 3H, -OCH3), $4.12\left(\mathrm{t}, \mathrm{J}=6.8 \mathrm{~Hz}, 2 \mathrm{H},-\mathrm{OCH}_{2}-\right), 4.06(\mathrm{~s}, 3 \mathrm{H},-\mathrm{OCH} 3), 3.99$ $(\mathrm{s}, 3 \mathrm{H},-\mathrm{OCH} 3), 3.25(\mathrm{t}, J=6.8 \mathrm{~Hz}, 2 \mathrm{H}, 5-\mathrm{H}), 2.04-2.08(\mathrm{~m}$, $\left.2 \mathrm{H},-\mathrm{CH}_{2}-\right), 1.79-1.83\left(\mathrm{~m}, 2 \mathrm{H},-\mathrm{CH}_{2}-\right) .{ }^{13} \mathrm{C} \mathrm{NMR}(100 \mathrm{MHz}$, $\left.\mathrm{CD}_{3} \mathrm{OD}\right) \delta: 152.9,152.2,151.9,151.0,146.3,145.6,139.7,135.2$, $134.7,129.9,128.0,124.5,123.2,121.3,120.5,113.3,110.0,69.9$, $62.6,57.6,57.3,57.0,50.2,28.0,27.8,26.7$.

2,9,10-Trimethoxy-3-(4-(4-methylpiperazin-1-yl)butoxy)-5,6dihydroisoquinolino[3,2-a]isoquinolin-7-ium (3b). Yellow solid; Yield: $43 \%$, m.p.: $195-196^{\circ} \mathrm{C}$ MS $(m / z) 492.2[\mathrm{M}]^{+},{ }^{1} \mathrm{H}$ NMR (400 MHz, CD 3 OD), $\delta: 9.76$ (s, $1 \mathrm{H}, 8-\mathrm{H}), 8.80$ (s, $1 \mathrm{H}$, $13-\mathrm{H}), 8.11(\mathrm{~d}, J=7.2 \mathrm{~Hz}, 1 \mathrm{H}, 11-\mathrm{H}), 8.02(\mathrm{~d}, J=7.2 \mathrm{~Hz}, 1 \mathrm{H}$, $12-\mathrm{H}), 7.66$ (s, 1H, 1-H), $7.03(\mathrm{~s}, 1 \mathrm{H}, 4-\mathrm{H}), 4.92(\mathrm{t}, J=6.8 \mathrm{~Hz}$, $2 \mathrm{H}, 6-\mathrm{H}), 4.20\left(\mathrm{~s}, 3 \mathrm{H},-\mathrm{OCH}_{3}\right), 4.15(\mathrm{t}, J=6.8 \mathrm{~Hz}, 2 \mathrm{H},-\mathrm{CH} 2)$, $4.10\left(\mathrm{~s}, 3 \mathrm{H},-\mathrm{OCH}_{3}\right), 3.99\left(\mathrm{~s}, 3 \mathrm{H},-\mathrm{OCH}_{3}\right), 3.30(\mathrm{t}, J=6.8 \mathrm{~Hz}$, $\left.2 \mathrm{H},-\mathrm{OCH}_{2}-\right), 3.26(\mathrm{t}, \mathrm{J}=6.8 \mathrm{~Hz}, 2 \mathrm{H}, 5-\mathrm{H}), 2.60-2.50(\mathrm{~m}$, $8 \mathrm{H}), 2.34\left(\mathrm{~s}, 3 \mathrm{H} \mathrm{N}-\mathrm{CH}_{3}\right), 1.84-1.91\left(\mathrm{~m}, 2 \mathrm{H},-\mathrm{CH}_{2}-\right), 1.71-1.79$ $\left(\mathrm{m}, 2 \mathrm{H},-\mathrm{CH}_{2}-\right) .{ }^{13} \mathrm{C}$ NMR $\left(100 \mathrm{MHz}, \mathrm{CD}_{3} \mathrm{OD}\right), \delta: 153.3$, $151.9,151.1,146.4,145.8,139.8,135.5,130.0,128.1,124.5,123.3$, $121.3,120.5,113.3,110.1,69.8,62.6,59.2,57.7,57.4,57.1,55.0$ (overlap2), 27.82, 27.77, 25.5 (overlap2), 24.1, 23.3.

2,9,10-Trimethoxy-3-(4-(piperidin-1-yl)butoxy)-5,6-dihydroisoquinolino[3,2-a]isoquinolin-7-ium Chloride (3c). Yellow solid; Yield: 52\%, m.p.: $197-198^{\circ} \mathrm{C}$. MS $(\mathrm{m} / z) 477.2[\mathrm{M}]^{+},{ }^{1} \mathrm{H}$
NMR (400 MHz, CD 3 OD) $\delta: 9.75$ (s, 1H, 8-H), 8.81 (s, $1 \mathrm{H}$, $13-\mathrm{H}), 8.11(\mathrm{~d}, J=7.2 \mathrm{~Hz}, 1 \mathrm{H}, 11-\mathrm{H}), 8.02(\mathrm{~d}, J=7.2 \mathrm{~Hz}, 1 \mathrm{H}$, $12-\mathrm{H}), 7.66$ (s, 1H, 1-H), $7.04(\mathrm{~s}, 1 \mathrm{H}, 4-\mathrm{H}), 4.92(\mathrm{t}, J=6.8 \mathrm{~Hz}$, $2 \mathrm{H}, 6-\mathrm{H}), 4.20(\mathrm{~s}, 3 \mathrm{H},-\mathrm{OCH} 3), 4.15\left(\mathrm{t}, \mathrm{J}=6.8 \mathrm{~Hz}, 2 \mathrm{H},-\mathrm{CH}_{2}\right)$, $4.10\left(\mathrm{~s}, 3 \mathrm{H},-\mathrm{OCH}_{3}\right), 3.99\left(\mathrm{~s}, 3 \mathrm{H},-\mathrm{OCH}_{3}\right), 3.27(\mathrm{t}, J=6.8 \mathrm{~Hz}$, 2H, 5-H), 2.78 (br, s, 6H), 1.86-1.89 (m, br, 4H), 1.71-1.74 (m, $4 \mathrm{H}), 1.58(\mathrm{~m}, 2 \mathrm{H}) .{ }^{13} \mathrm{C} \mathrm{NMR}\left(100 \mathrm{MHz}, \mathrm{CD}_{3} \mathrm{OD}\right) \delta: 153.0$, 151.9, 151.0, 146.3, 145.7, 139.8, 135.3, 130.0, 128.1, 124.5, 123.3, $121.3,120.5,113.3,110.1,70.0,62.6,59.2,57.7,57.4,57.1,55.0$ (overlap2), 27.82, 27.77, 25.5 (overlap2), 24.2, 23.3.

2,9,10-Trimethoxy-3-(4-morpholinobutoxy)-5,6-dihydroisoquinolino[3,2-a]isoquinolin-7-ium Chloride (3d). Yellow solid; Yield: $59 \%$, m.p. $200-201^{\circ} \mathrm{C}$. MS $(\mathrm{m} / z) 478.8[\mathrm{M}]^{+} .{ }^{1} \mathrm{H}$ NMR (400 MHz, CD 3 OD), $\delta: 9.75$ (s, $1 \mathrm{H}, 8-\mathrm{H}), 8.80$ (s, $1 \mathrm{H}$, $13-\mathrm{H}), 8.09$ (d, $J=7.2 \mathrm{~Hz}, 1 \mathrm{H}, 11-\mathrm{H}), 8.01$ (d, $J=7.2 \mathrm{~Hz}, 1 \mathrm{H}$, $12-\mathrm{H}), 7.64$ (s, 1H, 1-H), $7.02(\mathrm{~s}, 1 \mathrm{H}, 4-\mathrm{H}), 4.93(\mathrm{t}, J=6.8 \mathrm{~Hz}$, $2 \mathrm{H}, 6-\mathrm{H}), 4.20\left(\mathrm{~s}, 3 \mathrm{H},-\mathrm{OCH}_{3}\right), 4.14(\mathrm{t}, \mathrm{J}=6.8 \mathrm{~Hz}, 2 \mathrm{H}$, $\left.-\mathrm{CH}_{2}\right), 4.14(\mathrm{~s}, 3 \mathrm{H},-\mathrm{OCH} 3), 3.98\left(\mathrm{~s}, 3 \mathrm{H},-\mathrm{OCH}_{3}\right), 3.7$ (br, s, $4 \mathrm{H}), 3.29(\mathrm{t}, J=6.0 \mathrm{~Hz}, 2 \mathrm{H}, 5-\mathrm{H}), 2.51$ (br, s, $6 \mathrm{H}), 1.84-1.88$ (m, 2H), 1.72-1.76 (m, 2H). ${ }^{13} \mathrm{C}$ NMR (100 MHz, CD 3 OD) $\delta$ : 153.2, 151.9, 151.1, 146.3, 145.7, 139.8, 135.3, 130.0, 128.0, 124.5, $123.2,121.2$, 120.3, 113.2, 110.1, 70.0, 67.6 (overlap2), 62.5, 59.7, 57.6, 57.4, 57.0, 54.7 (overlap2), 28.1, 27.8, 23.8.

3-(4-(Diethylamino)butoxy)-2,9,10-trimethoxy-5,6-dihydroisoquinolino[3,2-a] isoquinolin-7-ium Chloride (3e). Yellow solid: Yield: $48 \%$, m.p.: $197-198^{\circ} \mathrm{C}$ MS $(m / z) 465.1[\mathrm{M}]^{+} .{ }^{1} \mathrm{H}$ NMR (400 MHz, CD 3 OD), $\delta: 9.75$ (s, 1H, 8-H), 8.80 (s, 1H, $13-\mathrm{H}), 8.09$ (d, $J=7.2 \mathrm{~Hz}, 1 \mathrm{H}, 11-\mathrm{H}), 8.01(\mathrm{~d}, J=7.2 \mathrm{~Hz}, 1 \mathrm{H}$, $12-\mathrm{H}), 7.64$ (s, 1H, 1-H), $7.02(\mathrm{~s}, 1 \mathrm{H}, 4-\mathrm{H}), 4.93(\mathrm{t}, J=6.8 \mathrm{~Hz}$, $2 \mathrm{H}, 6-\mathrm{H}), 4.20\left(\mathrm{~s}, 3 \mathrm{H},-\mathrm{OCH}_{3}\right), 4.12(\mathrm{t}, \mathrm{J}=6.8 \mathrm{~Hz}, 2 \mathrm{H}$, $\left.-\mathrm{CH}_{2}\right), 4.08\left(\mathrm{~s}, 3 \mathrm{H},-\mathrm{OCH}_{3}\right), 3.98\left(\mathrm{~s}, 3 \mathrm{H},-\mathrm{OCH}_{3}\right), 3.70$ (br, s, 
$4 \mathrm{H}), 3.29(\mathrm{t}, J=6.0 \mathrm{~Hz}, 2 \mathrm{H}, 5-\mathrm{H}), 2.51(\mathrm{br}, \mathrm{s}, 6 \mathrm{H}), 1.84-1.88$ $(\mathrm{m}, 2 \mathrm{H}), 1.72-1.76(\mathrm{~m}, 2 \mathrm{H}) .{ }^{13} \mathrm{C}$ NMR $\left(100 \mathrm{MHz}, \mathrm{CD}_{3} \mathrm{OD}\right) \delta$ : $153.2,151.9,151.1,146.3,145.7,139.8,135.3,130.0,128.0,124.5$, $123.2,121.2,120.3,113.2,110.1,70.0,67.6$ (overlap2), 62.5, 59.7, 57.6, 57.4, 57.0, 54.7 (overlap2), 28.1, 27.8, 23.8.

3-(4-Aminobutoxy)-2,9,10-trimethoxy-5,6-dihydroisoquinolino[3,2-a]isoquinolin-7-ium Chloride (3g). Yellow solid; Yield: 45\%, m.p.: $198-199^{\circ} \mathrm{C}$. MS $(m / z) 409.8[\mathrm{M}]^{+},{ }^{1} \mathrm{H}$ NMR (400 MHz, CD 3 OD) $\delta: 9.74(\mathrm{~s}, 1 \mathrm{H}, 8-\mathrm{H}), 8.80$ (s, 1H, 13-H), 8.09 (d, $J=7.2 \mathrm{~Hz}, 1 \mathrm{H}, 11-\mathrm{H}), 8.01(\mathrm{~d}, J=7.2 \mathrm{~Hz}, 1 \mathrm{H}, 12-\mathrm{H})$, $7.61(\mathrm{~s}, 1 \mathrm{H}, 1-\mathrm{H}), 7.01(\mathrm{~s}, 1 \mathrm{H}, 4-\mathrm{H}), 4.93(\mathrm{t}, \mathrm{J}=6.8 \mathrm{~Hz}, 2 \mathrm{H}$, $6-\mathrm{H}), 4.19\left(\mathrm{~s}, 3 \mathrm{H},-\mathrm{OCH}_{3}\right), 4.13\left(\mathrm{t}, \mathrm{J}=6.8 \mathrm{~Hz}, 2 \mathrm{H},-\mathrm{CH}_{2}\right)$, $4.07\left(\mathrm{~s}, 3 \mathrm{H},-\mathrm{OCH}_{3}\right), 3.98\left(\mathrm{~s}, 3 \mathrm{H},-\mathrm{OCH}_{3}\right), 3.64(\mathrm{t}, J=6.8 \mathrm{~Hz}$, $2 \mathrm{H}), 3.29$ (t, $J=6.8 \mathrm{~Hz}, 2 \mathrm{H}, 5-\mathrm{H}), 1.88-1.91(\mathrm{~m}, 2 \mathrm{H}), 1.71-1.75$ (m, 2H). ${ }^{13} \mathrm{C}$ NMR (100 MHz, $\left.\mathrm{CD}_{3} \mathrm{OD}\right) \delta: 153.3,151.9,151.1$, $146.3,145.7,139.8,135.2,130.0,128.0,124.5,123.2,121.2,120.3$, $113.2,110.2,70.1,62.6,57.6,57.4,57.1,54.7,30.2,27.8,26.8$.

3-(2-Hydroxyethoxy)-2,9,10-trimethoxy-5,6-dihydroisoquinolino[3,2-a]isoquinolin-7-ium Chloride (3f). Yellow solid. Yield: $41 \%$. m.p.: $219-220^{\circ} \mathrm{C}$, MS $(\mathrm{m} / z) 382.3[\mathrm{M}]^{+} .{ }^{1} \mathrm{H}$ NMR (400 MHz, CD 3 OD) $\delta: 9.76(\mathrm{~s}, 1 \mathrm{H}, 8-\mathrm{H}), 8.80$ (s, 1H, 13-H), $8.11(\mathrm{~d}, J=7.2 \mathrm{~Hz}, 1 \mathrm{H}, 11-\mathrm{H}), 8.02(\mathrm{~d}, J=7.2 \mathrm{~Hz}, 1 \mathrm{H}, 12-\mathrm{H})$, $7.68(\mathrm{~s}, 1 \mathrm{H}, 1-\mathrm{H}), 7.07(\mathrm{~s}, 1 \mathrm{H}, 4-\mathrm{H}), 4.92(\mathrm{t}, J=6.0 \mathrm{~Hz}, 2 \mathrm{H}$, $6-\mathrm{H}), 4.20\left(\mathrm{~s}, 3 \mathrm{H},-\mathrm{OCH}_{3}\right), 4.18\left(\mathrm{t}, \mathrm{J}=6.0 \mathrm{~Hz}, 2 \mathrm{H},-\mathrm{OCH}_{2}\right)$, $4.10\left(\mathrm{~s}, 3 \mathrm{H},-\mathrm{OCH}_{3}\right), 4.01\left(\mathrm{~s}, 3 \mathrm{H},-\mathrm{OCH}_{3}\right), 3.93(\mathrm{t}, J=6.0 \mathrm{~Hz}$, $\left.2 \mathrm{H},-\mathrm{OCH}_{2}-\right), 3.26(\mathrm{t}, \mathrm{J}=6.0 \mathrm{~Hz}, 2 \mathrm{H}, 5-\mathrm{H}) .{ }^{13} \mathrm{C} \mathrm{NMR}$ $\left(100 \mathrm{MHz}, \mathrm{CD}_{3} \mathrm{OD}\right) \delta: 153.1,151.9,151.1,146.4,145.76,139.8$, $135.3,130.1,128.1,124.5,123.3,121.3,120.7,113.5,110.2,71.9$, $62.5,61.5,57.7,57.4,57.1,27.8$.

\section{Biological Activity}

4.1. In Vitro Inhibition Studies of AChE and BuChE. Acetylcholinesterase (AChE, from electric eel), butyrylcholinesterase (BuChE, from equine serum), 5,5' -dithiobis-(2-nitrobenzoic acid) (Ellman's reagent, DTNB), acetylthiocholine chloride (ATC), and butyrylthiocholine chloride (BTC) were got from Sigma Aldrich. Jatrorrhizine derivatives were dissolved in DMSO and then diluted in $0.1 \mathrm{M} \mathrm{KH}_{2} \mathrm{PO} 4 / \mathrm{K}_{2} \mathrm{HPO} 4$ buffer ( $\mathrm{pH} 8.0$ ) to provide a final concentration range.

All the assays were carried out under $0.1 \mathrm{M} \mathrm{KH}_{2} \mathrm{PO}_{4} /$ $\mathrm{K}_{2} \mathrm{HPO}_{4}$ buffer, $\mathrm{pH}$ 8.0, using a Shimadzu UV-2450 Spectrophotometer. AChE and BuChE solutions were prepared to give 2.0 units $/ \mathrm{mL}$ in $2 \mathrm{~mL}$ aliquots. The assay medium $(1 \mathrm{~mL})$ consisted of phosphate buffer $(\mathrm{pH} 8.0), 50 \mu \mathrm{L}$ of $0.01 \mathrm{M}$ DTNB, $10 \mu \mathrm{L}$ of enzyme, and $50 \mu \mathrm{L}$ of $0.01 \mathrm{M}$ substrate (ACh chloride solution). Test compounds were added to the assay solution and preincubated at $37^{\circ} \mathrm{C}$ with the enzyme for $15 \mathrm{~min}$ followed by the addition of substrate. The activity was determined by measuring the increase in absorbance at $412 \mathrm{~nm}$ at $1 \mathrm{~min}$ intervals at $37^{\circ} \mathrm{C}$. Calculations were performed according to the method of the equation of Suzuki et al. $[18,19]$. Each concentration was assayed in triplicate. In vitro $\mathrm{BuChE}$ assay was similar to the method described above. The $\mathrm{IC}_{50}$ values for $\mathrm{AChE}$ and $\mathrm{BuChE}$ inhibition are shown in Table 1 .

\section{Molecular Modeling Studies}

To determine the possible mode of reaction between compounds and $T$. californica enzyme (TcAChE), one of the jatrorrhizine derivatives was docked to the AChE active site gorge using the Autodock Vina software [20], based on the structure of the complex of TcAChE (PDB entry 5FUM) [21]. The most probable conformations of the ligands were chosen based on the docked energy value. The position of compound $3 \mathbf{g}$ in the binding site with respect to the key residues is shown in Figures 2(a) and 2(b). In TcAChE, the amino acid residues Tyr72, Tyr124, Tyr337, Tyr341, Asp74, Trp86, Trp286, Gly120, Ser293, and Phe338 are in hydrophobic contact with compound $\mathbf{3 g}$, and Tyr133 and Glu202 are shown to be involved in hydrogen-bonding interactions with molecule $3 \mathrm{~g}$. These interactions are significant and might explain the high affinity of the compound $3 \mathrm{~g}$ to TcAChE (Figure 2).

\section{Conclusion}

In conclusion, a series of jatrorrhizine derivatives with substituted amino groups linked at the 3-position were designed, synthesized, and biologically evaluated as inhibitors of acetylcholinesterase. All these jatrorrhizine derivatives were proved to be potent inhibitors of acetylcholinesterase (AChE) with submicromolar $\mathrm{IC}_{50}$ values, but less sensitive to butyrylcholinesterase (BuChE), which suggests that these jatrorrhizine derivatives are selective for $\mathrm{AChE} / \mathrm{BuChE}$. Compound $3 \mathrm{~g}$ gave the most potent inhibitor activity for AChE $\left(\mathrm{IC}_{50}=0.301 \mu \mathrm{M}\right)$, which is greater than the lead compound jatrorrhizine. All these results demonstrated that these jatrorrhizine derivatives are potential inhibitors for Alzheimer's disease $(\mathrm{AD})$.

\section{Abbreviations}

AchE: Acetylcholinesterase

BuChE: Butyrylcholinesterase

Ach: Acetylcholine

NMR: Nuclear magnetic resonance spectroscopy

MS: $\quad$ Mass spectrometer.

\section{Conflicts of Interest}

The authors declare that there are no conflicts of interest regarding the publication of this paper.

\section{Acknowledgments}

This work was supported by the Guizhou Province Natural Science Foundation ([2015]2123) (3001433), Chongqing Education Commission Fund [KJ131120], Natural Science Foundation of Guangdong Province (2016A010105015), Strategic Resources Service Network Program on Plant Genetic Resources Innovation of the Chinese Academy of Sciences (no. ZSZC-005), and the Guangdong Province Science \& Technology Project [Grant no. 2016A010105015]. 


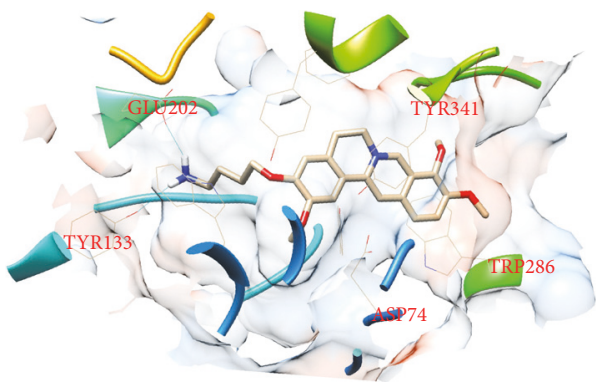

(a)

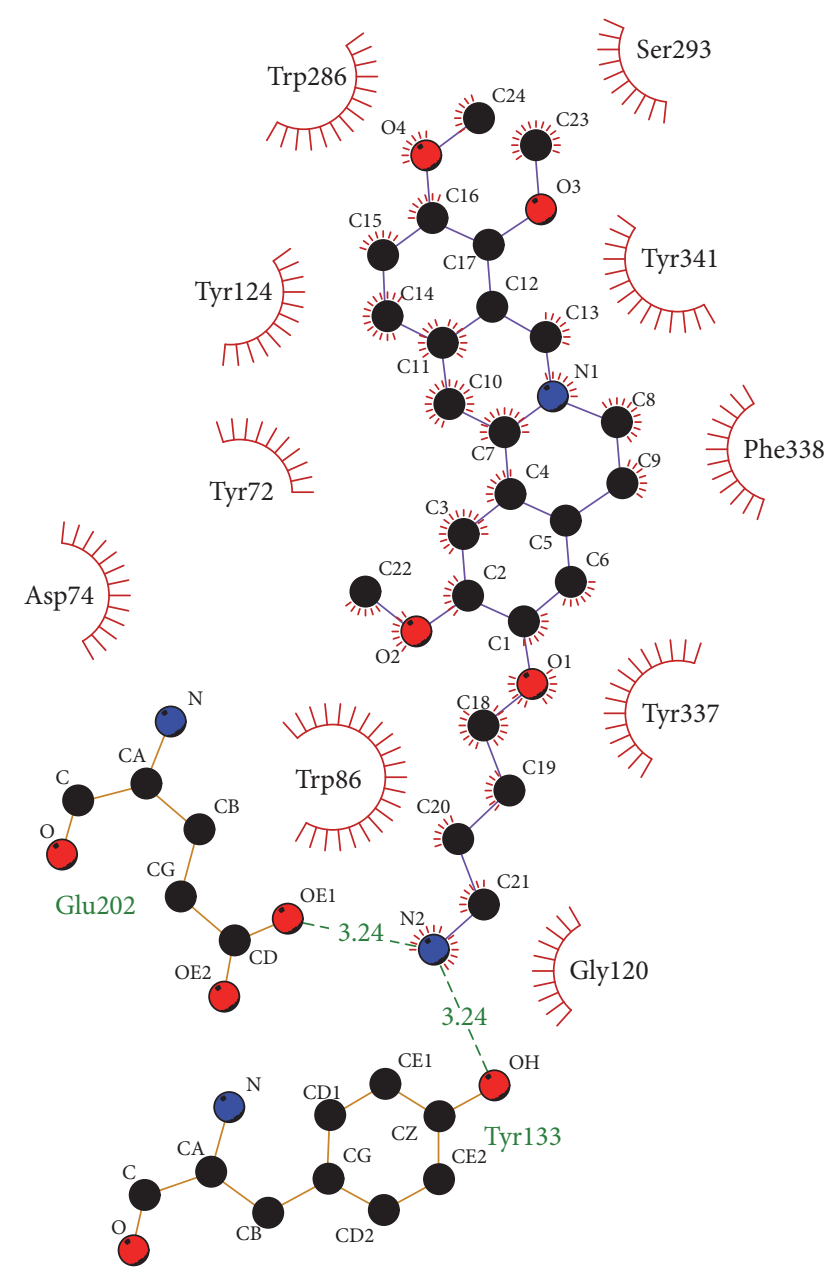

(b)

Figure 2: Docking models of the compound-enzyme complex. (a) Stereoviews looking down the gorge of TcAChE binding with 3g; (b) representation of compound $\mathbf{3 g}$ docked into the binding site of $\mathrm{AChE}$ highlighting the protein residues that form the main interactions with the inhibitor. Hydrogen-bonding interaction between ligand and residues Tyr133 and Glu202 is shown with the green line. The residues of TcAChE involved in hydrogen-bonding and hydrophobic interactions with molecule $\mathbf{3 g}$ were analyzed using Chimera 1.9 [13] and Ligplot ${ }^{+}$ [14].

\section{Supplementary Materials}

Supplementary data (ESI-MS, ${ }^{1} \mathrm{H}$ NMR, and ${ }^{13} \mathrm{C}$ NMR) associated with this article can be found in the online version. (Supplementary Materials)

\section{References}

[1] People's Daily, http://neuro.dxy.cn/article/74119.

[2] V. N. Talesa, "Acetylcholinesterase in Alzheimer's disease," Mechanisms of Ageing \& Development, vol. 122, no. 16, pp. 19611969, 2001.

[3] T. Friedemann, U. Schumacher, Y. Tao, A. K. Leung, and S. Schröder, "Neuroprotective activity of coptisine from," Evidence-Based Complementary and Alternative Medicine, vol. 2015, Article ID 827308, 9 pages, 2015.

[4] H. Zhao, S. Zhou, M. Zhang et al., "An in vitro AChE inhibition assay combined with UF-HPLC-ESI-Q-TOF/MS approach for screening and characterizing of $\mathrm{AChE}$ inhibitors from roots of Coptis chinensis Franch," Journal of Pharmaceutical \& Biomedical Analysis, vol. 120, pp. 235-240, 2015.

[5] L. Yuan, D. Tu, X. Ye, and J. Wu, "Hypoglycemic and hypocholesterolemic effects of Coptis chinensis Franch inflorescence," Plant Foods for Human Nutrition, vol. 61, no. 3, pp. 139-144, 2006.

[6] L. Grycováa, J. Dostálc, and R. Marek, "Quaternary protoberberine alkaloids," Phytochemistry, vol. 68, no. 2, pp. 150-175, 2007.

[7] F. U. Yan, B. R. Hu, Q. Tang, F. U. Qin, Q. Y. Zhang, and J. Z. Xiang, "Effect of jatrorrhizine, berberine, Huanglian Decoction and compound-mimic prescription on blood glucose in mice," Chinese Traditional \& Herbal Drugs, vol. 38, no. 4, pp. 548-551, 2006.

[8] Y. Yu, Z. Yi, and Y. Liang, "Main antimicrobial components of Tinospora capillipes, and their mode of action against Staphylococcus aureus," FEBS Letters, vol. 581, no. 22, pp. 41794183, 2007. 
[9] L. Račková, M. Májeková, D. Košt’álová, and M. Štefek, "Antiradical and antioxidant activities of alkaloids isolated from Mahonia aquifolium structural aspects," Bioorganic \& Medicinal Chemistry, vol. 12, no. 17, pp. 4709-4715, 2004.

[10] L. Huang, A. Shi, F. He, and X. Li, "Synthesis, biological evaluation, and molecular modeling of berberine derivatives as potent acetylcholinesterase inhibitors," Bioorganic \& Medicinal Chemistry, vol. 18, no. 3, pp. 1244-1251, 2010.

[11] A. Shi, L. Huang, C. Lu, F. He, and X. Li, "Synthesis, biological evaluation and molecular modeling of novel triazole-containing berberine derivatives as acetylcholinesterase and $\beta$-amyloid aggregation inhibitors," Bioorganic \& Medicinal Chemistry, vol. 19, no. 7, pp. 2298-2305, 2011.

[12] T. Su, S. Xie, H. J. Wei, L. Huang, and X. Li, "Synthesis and biological evaluation of berberine-thiophenyl hybrids as multifunctional agents: Inhibition of acetylcholinesterase, butyrylcholinesterase, and A $\beta$ aggregation and antioxidant activity," Bioorganic \& Medicinal Chemistry, vol. 21, no. 18, pp. 58305840, 2013.

[13] E. F. Pettersen, T. D. Goddard, C. C. Huang et al., "UCSF Chimera-a visualization system for exploratory research and analysis," Journal of Computational Chemistry, vol. 25, no. 13, pp. 1605-1612, 2004.

[14] A. C. Wallace, R. A. Laskowski, and J. M. Thornton, "LIGPLOT: a program to generate schematic diagrams of protein-ligand interactions," Protein Engineering, Design and Selection, vol. 8, no. 2, pp. 127-134, 1995.

[15] L. Wang, X. Ye, Z. Chen et al., "Synthesis and antimicrobial activity of 3-octyloxy-8-alkyljatrorrhizine derivatives," Journal of Asian Natural Products Research, vol. 11, no. 4, pp. 365-370, 2009.

[16] Y. M. Feng and K. M. Xun, "Extraction of jatrorrhizine chloride fromthe mother liquid of producing berberine chloride," China Journal of Chinese Materia Medica, vol. 14, no. 1, pp. 29-30, 1989.

[17] J. Y. Pang, Y. Qin, W. H. Chen, G. A. Luo, and Z. H. Jiang, "Synthesis and DNA-binding affinities of monomodified berberines," Bioorganic \& Medicinal Chemistry, vol. 13, no. 20, pp. 5835-5840, 2005.

[18] N. Suzuki, T. T. Cheung, X.-D. Cai et al., "An increased percentage of long amyloid beta protein secreted by familial amyloid beta protein precursor (beta APP717) mutants," Science, vol. 264, no. 5163, pp. 1336-1340, 1994.

[19] K. N. Dahlgren, A. M. Manelli, W. Blaine Stine Jr., L. K. Baker, G. A. Krafft, and M. J. Ladu, "Oligomeric and fibrillar species of amyloid- $\beta$ peptides differentially affect neuronal viability," The Journal of Biological Chemistry, vol. 277, no. 35, pp. 3204632053, 2002.

[20] O. Trott and A. J. Olson, "AutoDock Vina: improving the speed and accuracy of docking with a new scoring function, efficient optimization and multithreading," Journal of Computational Chemistry, vol. 31, no. 2, pp. 455-461, 2010.

[21] C. Engdahl, S. Knutsson, F. Ekström, and A. Linusson, "Discovery of selective inhibitors targeting acetylcholinesterase 1 from disease-transmitting mosquitoes," Journal of Medicinal Chemistry, vol. 59, no. 20, pp. 9409-9421, 2016. 

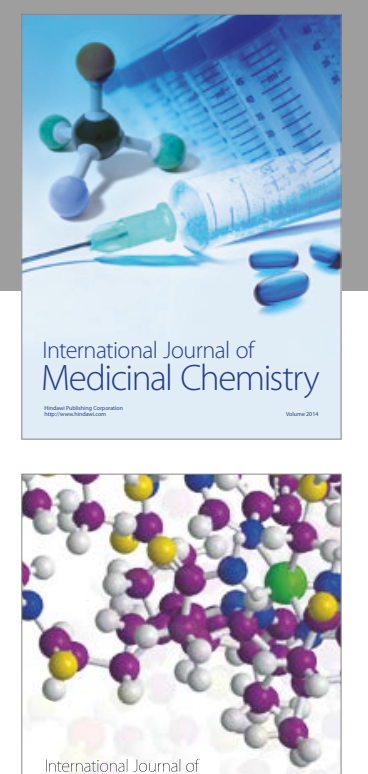

Carbohydrate Chemistry

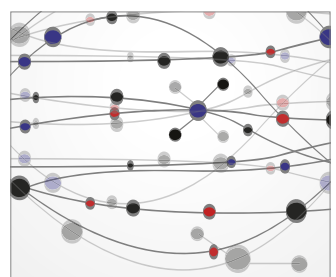

The Scientific World Journal
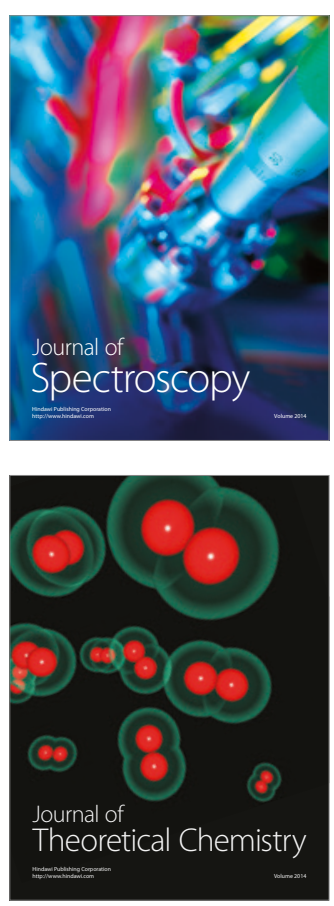
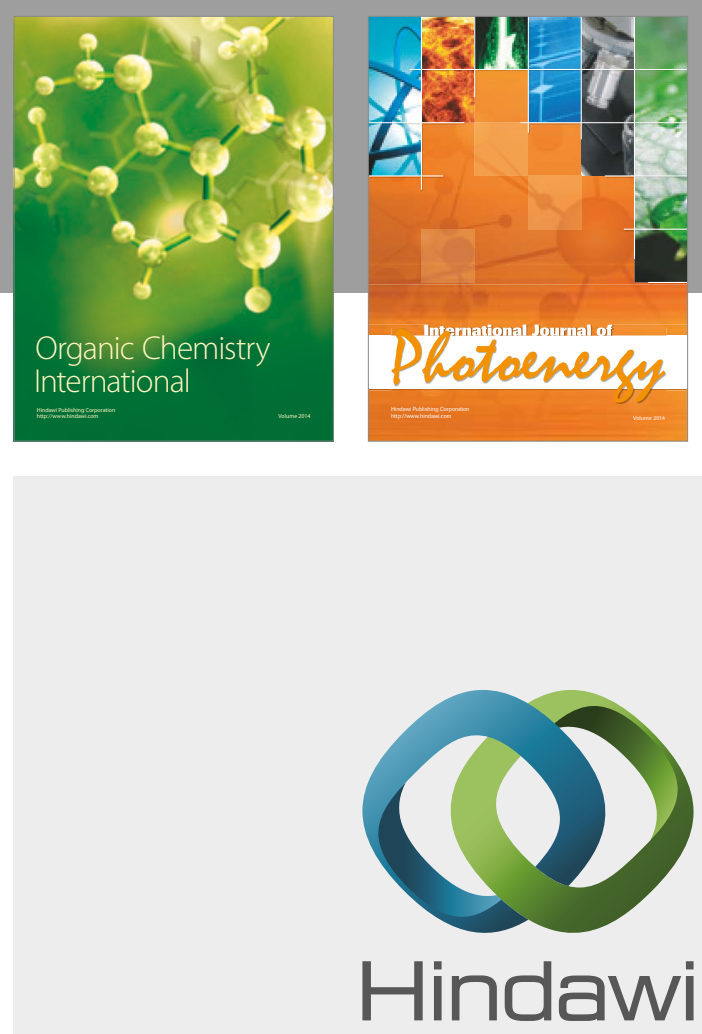

Submit your manuscripts at

https://www.hindawi.com

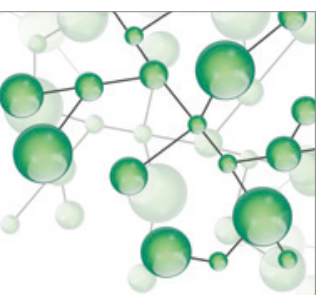

International Journal of

Inorganic Chemistry

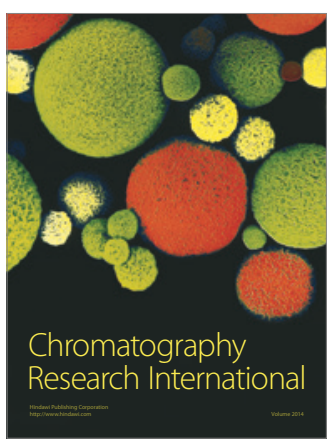

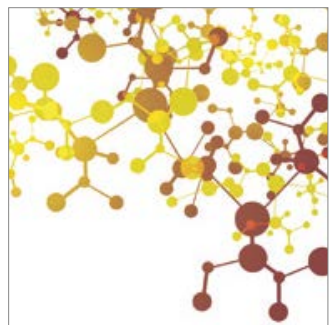

Applied Chemistry
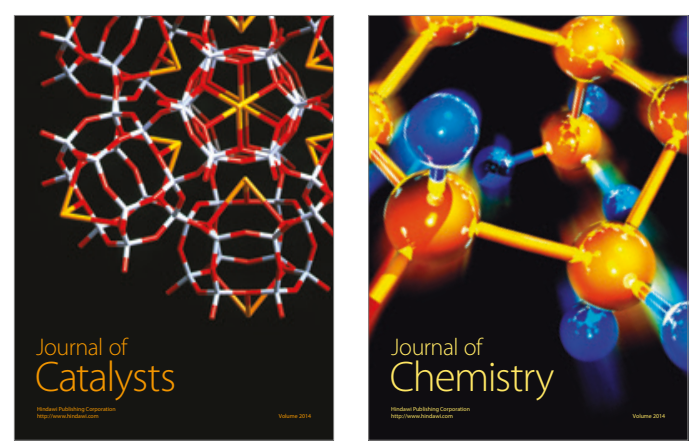
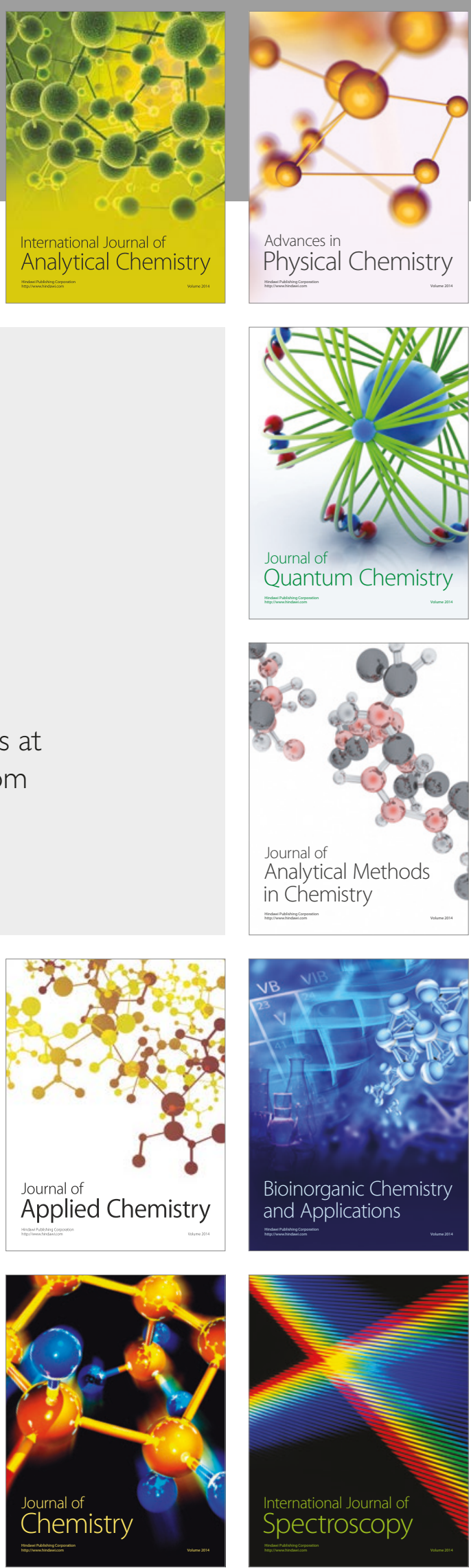